- ACORN Australan colege of | JOURNAL OF PERIOPERATIVE NURSING

Volume 33 | Issue 2

Article 5

$6-1-2020$

\title{
Perioperative nurses' engagement with the surgical safety checklist: A focused ethnography
}

Follow this and additional works at: https://www.journal.acorn.org.au/jpn

Part of the Health Services Administration Commons, Health Services Research Commons, Perioperative, Operating Room and Surgical Nursing Commons, and the Surgery Commons

(c) (1)

This work is licensed under a Creative Commons Attribution 4.0 License.

\section{Recommended Citation}

Rogers, Julie A. Mrs; Mcleish, Paul Mr; and Alderman, Jan Mrs (2020) "Perioperative nurses' engagement with the surgical safety checklist: A focused ethnography," Journal of Perioperative Nursing: Vol. 33 : Iss. 2 , Article 5.

Available at: https://doi.org/10.26550/2209-1092.1066

https://www.journal.acorn.org.au/jpn/vol33/iss2/5

This Article is brought to you for free and open access by Journal of Perioperative Nursing. It has been accepted for inclusion in Journal of Perioperative Nursing by an authorized editor of Journal of Perioperative Nursing. 


\section{Perioperative nurses' engagement with the surgical safety checklist: A focused ethnography}

\section{Cover Page Footnote}

Acknowledgements This work was submitted to the University of Adelaide as a requirement for completion of a master's degree, subject 7021d. The author would like to acknowledge their research supervisors, Paul McCleish and Jan Alderman, and the unit co-ordinator, Phillipa Rasmussen, for their ongoing encouragement and support in writing this article. The author also expresses their gratitude to the operating room nurses who participated in this study. 


\section{Authors}

Julie Rogers

MNSc, BN, GDip Periop

Clinical Nurse, University of Adelaide

Paul McLeish

MNSc, BN, GDip Orth, PhD candidate

Lecturer and Course Coordinator Adelaide

Nursing School, University of Adelaide

\section{Jan Alderman}

BN, GDip NSc, MNSC

Lecturer and Course Coordinator Adelaide Nursing School, University of Adelaide

\section{Corresponding author}

Julie Rogers

BN, GDip Periop

Clinical Nurse, University of Adelaide julie.rogers2@sa.gov.au

\section{Perioperative nurses' engagement with the surgical safety checklist: A focused ethnography}

Abstract

\section{Purpose}

To gain greater insight into how nurses engage with the multidisciplinary team during the surgical safety checklist process.

\section{Participants and setting}

Participants were a purposeful sample of eight operating room nurses. The study was conducted in the operating room department of a major tertiary teaching hospital in South Australia.

\section{Methods}

Phase 1 employed participant observations while phase 2 employed semistructured interviews.

\section{Findings}

Participants supported the use of the surgical safety checklist and valued its role to enhance patient safety. Multidisciplinary team culture played a significant role in how the checklist was conducted and heavily influenced the level of nurse participation in the process. Observations indicated poor compliance with the nursing review section of the checklist.

\section{Conclusions}

Multidisciplinary team engagement is important for effective communication during the process of utilising the surgical safety checklist to promote safe outcomes for patients. Nurses are more likely to actively participate in the checklist process if they feel their role is valued by other team members. The nurse-specific area of the checklist is an area for improvement as this tends to be overlooked and considered less important than other checklist items. Modifications in timing the checklist and nurse training may be beneficial to address non-compliance with the nurse area of the checklist.

Keywords: surgical safety checklist, team time out, perioperative nursing, teamwork, patient safety, adverse events, teamwork, preventable errors, operating room.

\section{Introduction}

Every year, an estimated 234 million surgical procedures are performed worldwide 1 . Evidence suggests that severe adverse events occur in approximately 3.6 per cent of all hospital admissions and root cause analysis indicates that 41 per cent of these errors are preventable ${ }^{2}$. Preventable errors - for example, wrong site, wrong patient and wrong procedure performed - can have devastating consequences for surgical patients ${ }^{3}$. In an effort to address the incidence of these errors the World Health Organization 
(WHO) published the Surgical Safety Checklist (SSC) in 2008. The checklist is a safety tool developed after extensive multinational and multidisciplinary consultation with surgeons, anaesthetists and operating room nurses and aims to prevent surgical errors and increase safety for patients undergoing surgery 4 . The SSC is a structured communication process in which all operating room staff present are required to participate prior to commencement of surgery. This final check prior to surgery aims to promote the sharing of all critical information, and allow potential errors or risks to be identified and mitigated $^{3}$. Compliance with the SCC has been shown to reduce the incidence of surgical errors and is recommended as an effective tool for improving teamwork and communication within the operating room ${ }^{5}$.

\section{Standards for Perioperative Nursing} in Australia, published by Australian College of Perioperative Nurses (ACORN), and the WHO SSC checklist have the expectation that all perioperative nurses contribute to patient safety by participating in the SSC process. This involves nurses engaging with the multidisciplinary team in verifying the correct patient, site and procedure to be performed. An additional section of the SSC requires nurses to confirm with the surgical team that all surgical instrumentation has undergone sterilisation and verify that the appropriate equipment is available for the procedure?. This aspect of the SSC is important in preventing the use of contaminated instruments, which is associated with surgical site infections, and ensuring all relevant equipment is available to prevent intra-operative delays ${ }^{3}$.

There have been numerous studies undertaken of the WHO safety checklist since its worldwide introduction in 2009. Research

conducted in the Australian

setting indicates compliance and participation in the SSC varies, and barriers to nurse involvement in the process include work pressures, workplace culture and time restraints ${ }^{7-9}$. There is limited information on how Australian nurses engage in the nurse-specific section of the SSC, and further research is needed to understand this area of operating room nursing practice.

\section{Purpose}

The purpose of this study was to gain a greater insight into how operating room nurses engage with the multidisciplinary team during the SSC, to explore any barriers that impede nurses from actively participating and compare the findings to previous research. The study was also an opportunity to address the gap in knowledge about how nurses use the nurse-specific section of the checklist in their practice.

\section{Participants and setting}

The study was conducted within the operating room department of a major South Australian public hospital with over forty operating rooms used for a variety of surgical specialties including trauma and elective surgery. The primary researcher was familiar with the environment and study participants. Observations were conducted randomly throughout the department. A purposive sample of eight nurses across a diverse range of surgical specialties and of various age groups were recruited for phase two interviews. To ensure interview participants had enough perioperative nursing experience and depth of knowledge of the research subject, only full- or parttime registered and enrolled nurses working within the operating room with 12 months of operating room nurse experience were included in the study.

\section{Methods}

The research was conducted as a focused ethnography as this method enables researchers to gain deeper insights into the behaviours and interactions of a group of people ${ }^{10}$. As the primary researcher was already part of the group being studied, this method of enquiry enabled them to bring their own insider (emic) view to the research, as well being able to adopt an outsider (etic) view to gain fresh insights into perioperative nursing practice ${ }^{11}$.

Data was collected across two distinct phases. The first phase involved non-participant observations of operating room staff including nurses, surgeons and anaesthetists prior to the commencement of each surgical case. Through observations the researcher was able to gain an understanding of the nursing practices and multidisciplinary team behaviours and communications that occurred during the SSC. The second phase of the study involved semistructured interviews with operating room nurses. The study design enabled the researcher to explore the observational findings identified from phase 1 and gain deeper insight into these findings through questioning key informants within the clinical setting in phase 2 .

\section{Data collection}

Ethical approval for the study was obtained by the appropriate human research ethics committee (HREC/18/CALHN/711). Permission to conduct the research was approved by the department's medical and nursing directors. Written consent for operating room staff observed was not obtained as observations were overt and operating rooms were randomly chosen for convenience. All operating room staff were aware 
they were being observed while the SSC was conducted and gave verbal consent to participate. A fact sheet was provided to staff regarding the purpose of the study to observe the interactions of perioperative nurses with the multidisciplinary team and assurances that no identifying information was to be recorded. All interview participants gave written consent prior to interviews and were provided with an information sheet explaining the aims of the research, that they were under no obligation to participate and that their anonymity would be maintained through use of pseudonyms.

Pilot observations were undertaken by the primary researcher to identify patterns of behaviour and areas appropriate for more systemic observation such as noise levels, the physical location of staff and whether nurses were actively participating ${ }^{12}$. In this early stage of research, the primary researcher noticed that the location of staff in the room and the direction that they faced were important indicators of how the checklist was being conducted. Following the recommendations made by Roper and Shapira' ${ }^{12}$ to describe the 'set' of the study, a basic map of the patient on the operating table, including locations and activities of all staff, was recorded in the observational field notes. A checklist was used to capture areas of interest such as which staff member initiated the SSC, noise levels, nurse participation, and whether nurses used the nursespecific area of the checklist so that the researcher could gain deeper understanding of the behaviours and group dynamics while the SSC was being conducted. The researcher used a journal to reflect upon their observational findings. Observations during phase 1 continued until no new insights were gained or new information collected in the setting

\section{Theme: Nurses perception of their role in the checklist}

\begin{tabular}{l|l|l|}
\hline Example from field note & Initial code & $\begin{array}{l}\text { Category/ } \\
\text { subtheme }\end{array}$ \\
\hline $\begin{array}{l}\text { Surgeon did not ask for nurse } \\
\text { feedback. }\end{array}$ & $\begin{array}{l}\text { Lack of } \\
\text { opportunity } \\
\text { to speak }\end{array}$ & $\begin{array}{l}\text { Surgeon } \\
\text { requesting nurse } \\
\text { feedback }\end{array}$ \\
$\begin{array}{l}\text { time out and nurses did not offer any } \\
\text { information. }\end{array}$ & \\
\hline
\end{tabular}

\section{Extracts from interviews}

'Being a senior nurse, I have no qualms about mentioning anything of concern to me.'

\section{Participant 5}

'I have a very good working relationship with the team and feel comfortable participating.' Participant 2

'I initiate the process with surgeons by reminding them and ensure that everyone is there to participate.'

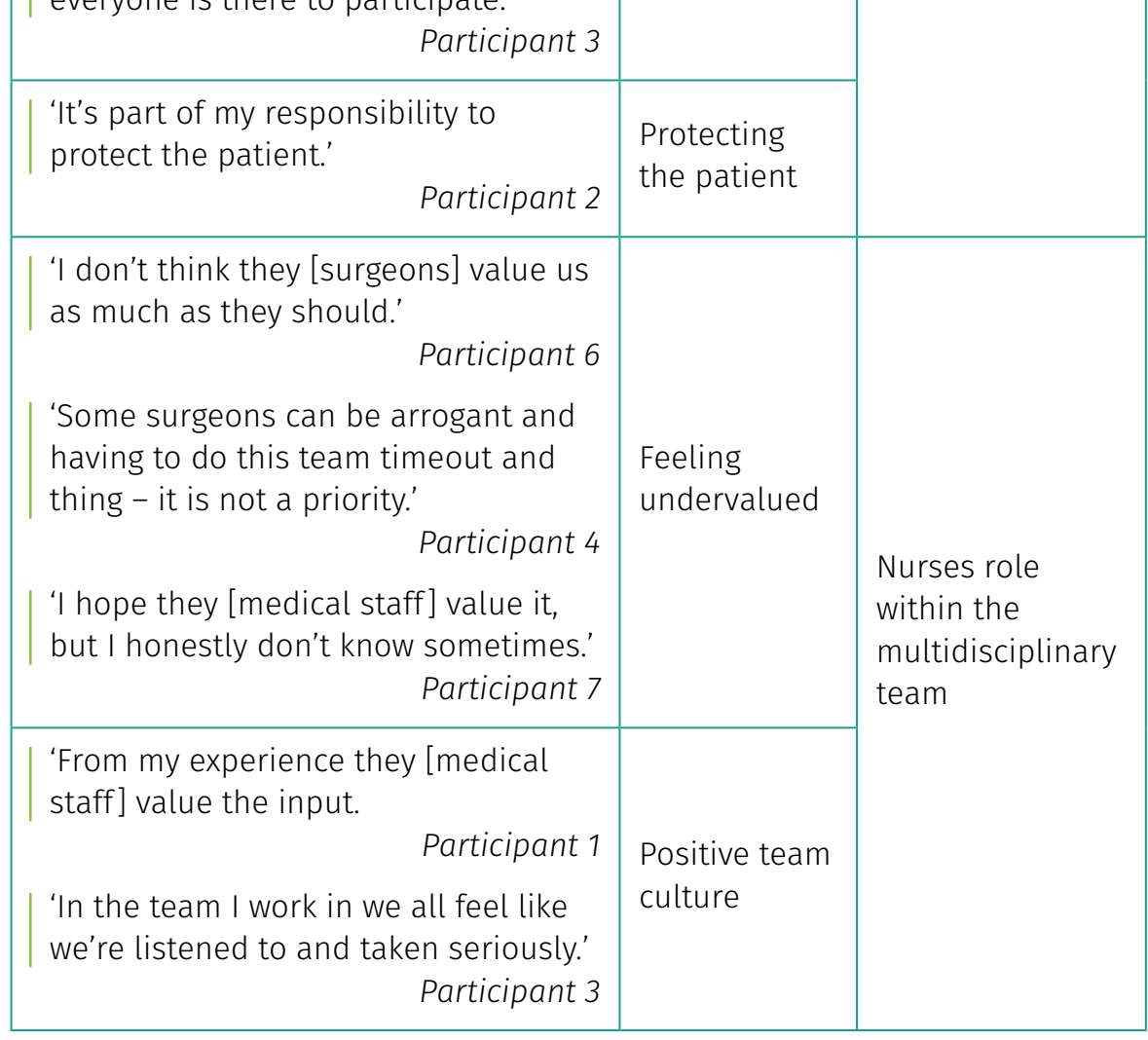

Figure 1: Example of data analysis speaking up

Nurses as patient safety advocates 
with the researcher concluding that they had reached the point of data saturation.

After observations from phase 1, eight semi-structured interviews were conducted over a period of two weeks. Interviews lasted approximately ten minutes, were undertaken at a mutually convenient location and time, and were audio recorded and transcribed verbatim. Open-ended questions were used to encourage participants to freely discuss their experiences with the SSC and to gain a deeper understanding of the observational findings. After eight interviews the researcher noticed similar themes and ideas were being reported from participants, and the researcher decided enough data had been obtained for analysis.

\section{Data analysis}

To analyse the data from observations and interviews the primary researcher used Braun and Clarke's framework for thematic data analysis $^{13}$. Data from phase 1 was reviewed multiple times to familiarise the researcher with the material and to allow the researcher to search for meanings and patterns. To further explore subthemes identified from phase 1 of the study the primary researcher incorporated these findings into the semi-structured interview questions to gain a deeper understating of these themes.

Phase 2 interview recordings were transcribed verbatim by the primary researcher and returned to participants for confirmation. Transcripts were re-read multiple times, carefully considered for meaning and initial codes were generated. Codes were compared with the observational data analysis to identify any similarities or variances and grouped into subthemes. The final step of data analysis involved a final refinement and regrouping of subthemes into three major themes. An example of the process of data analysis from phases 1 and 2 of the research from initial coding to subthemes and then to form an overarching theme is illustrated in Figure 1.

\section{Rigour}

To enhance the trustworthiness and credibility of this study the primary researcher used the methods of prolonged engagement with the data, triangulation and peer debriefing with their academic supervisors. Member checks with interview participants were undertaken to ensure that the interpretations remained true to the participants' experiences $^{14,15}$. Reflexivity of the research process was maintained with the use of a reflective journal to demonstrate transparency and minimise bias ${ }^{10}$. To promote confirmability and completeness of the research, the primary researcher triangulated two different data sources (observations and interviews) to investigate the issue being studied to gain a deeper and more meaningful understanding of the data ${ }^{14}$.

\section{Findings}

The findings of this study were analysed from observational data collected from observing conduction of twenty SSCs and interview data obtained from eight operating room nurses of various age groups and experience levels. Three main themes were identified from data analysis:

1. varied nurse participation in conducting the checklist

2. the influence of team culture, leadership and expectations on conducting the checklist

3. nurses' perception of their role in conducting the checklist.
Theme 1: Varied nurse participation in the checklist

Nursing participation in conducting the SSC emerged as a theme as the observational and interview findings identified significant inconsistencies in how nurses participated in this activity. Observational findings indicated variety in nurse behaviours and communication within the multidisciplinary team and interview participants described different beliefs about how and why nurses should participate while the SSC is conducted.

Although the department policy recommends that the senior surgeon performs the checklist, the researcher observed and nurses identified in the interviews that they or other nurses initiated the checklist process by prompting the multidisciplinary team to start the checklist and by organising checklist paperwork in advance. Interview participants described the checklist as a priority and were confident that it was an important safety tool, and not simply another process that needed to be followed.

One participant described the checklist as:

\section{'... important for patient safety and I make it a priority to be involved'.}

Participant 3

In every observation, at least one nurse in the operating room participated with the multidisciplinary team during the checking process by standing and remaining with the patient to confirm identification, consent and procedure with the surgeon and anaesthetist. One participant explained that it 
was important for all nurses in the operating room:

'... to be present, not only in body but to focus and concentrate on the checklist'.

Participant 8

Although the majority of nurses observed appeared to support the use of the checklist, there were variations in how the nursing team participated. Nurses tended to nominate themselves or another nurse to represent the nursing team to 'do the time out' in an ad hoc fashion. This contradicts the WHO guidelines and department policy which recommend full team participation in conducting the checklist. This trend varied throughout the observations. The primary researcher observed instances where while one nurse participated, others would be performing other tasks such as documentation, locating and setting up equipment and conversing with other staff members. This finding was discussed during the interviews and there was a variety of views about whether all nurses present should be involved in the time out process. Some participants felt not all nurses need be involved.

'I think only one nurse needs to be involved, it's probably not necessary for all of us to be there.'

Participant 2

Another noted:

'I actually think not all scrub staff need to participate, but we should all be listening in'.

\section{Participant 8}

Other respondents strongly believed all nurses in the room should be involved in conducting the checklist to prevent communication failures.
'I believe the entire team should be involved as not all nurses stay in the room at one time and to make sure no information is lost.'

\section{Participant 2}

This variation in practice behaviours suggests vastly different beliefs about if and how nurses should be involved, and contradicts the existing policy and expectation that the full nursing team should participate during the checklist.

At no time was it observed that all nurses fully engaged in the process recommended by ACORN and WHO to confirm instrument sterility with the multidisciplinary team. Furthermore, at the time the SCC was conducted no instrument trays were open for staff to check the sterile indicators. Information was infrequently offered by nurses to surgeons regarding any concerns or equipment. In one observation, surgical implants were verified between the nursing staff and surgeon and in another the use of an energy device was confirmed between nursing staff and surgeon. In many observations the surgeons did not ask the nurses whether they had any concerns which indicates that this area of the checklist was frequently overlooked.

When interview participants were asked if they confirmed instrument sterility and equipment availability there seemed to be confusion about the question, which may indicate a lack of awareness of this part area of the checklist. One participant disagreed with this part of the checklist as they felt that it was inappropriate.

'Technically, it's not really safe to be opening instruments during time out as there is a lot of movement in the theatre during the time which could contaminate instruments.'

Participant 6
Other respondents explained that the instruments aren't opened at the time when the SSC is conducted as it occurs when the patient has been brought into the room and instruments trays are not opened at that time.

'As a rule, the instruments aren't open until after the checklist, it's a timing issue'

Participant 4

Theme 2: The influence of team culture, leadership and expectations on conducting the checklist

This theme represented the noticeable differences in multidisciplinary team dynamics across the department and the resulting influence on communication and behaviour while the SSC was conducted.

In all observations the checklist was undertaken when the patient first arrived in the operating room. The preoperative items on the SSC were completed in one phase before the patient was anaesthetised rather than the two-phase approach that is recommended by the WHO. The primary researcher observed noticeable differences in how members of the operating room team interacted with each other while the SSC was conducted. It was obvious from some of the observations that there was ineffective or poor compliance with the local policy. In those observations where the multidisciplinary team did 'come together' the whole team, including nurses, surgeons and anaesthetists, congregated around the patient and all other workflow activities ceased while the SSC was conducted. Everyone in the room appeared to share a common understanding that the checklist needed to be completed and noise levels in the room were lower as other tasks 
were put on hold. The physical location of staff during the process recorded on the observational field notes demonstrate that when staff faced towards each other and stood around the patient, greater levels of information were shared and there seemed to be a strong sense of group unity.

In other observations there was limited team engagement and less communication between team members from all disciplines. During some observations the person who led the checklist (often the surgeon) had to prompt people several times that the time out needed to be done and, even then, not all staff in the room would participate. Staff who declined to take place seemed disinterested in the process and performed other work-related tasks including talking to others, moving equipment, walking in and out of the room and accessing computers. Some of the other activities such as conversations and setting up equipment contributed to background noise in those rooms at that time. This appeared to be accepted by and normalised by those present, as no one was reminded to pause and participate, which seems to indicate noise and distractions while conducting the SSC were acceptable. Differences in the level of engagement across disciplines and by all staff present are suggestive of a wide variation in culture and associated practice.

Existing differences in team cultures was also reflected in interview responses. One participant reported:

'We need better whole theatre participation and I have only ever seen it a couple of times in my whole life. Some people [other staff members] don't seem to understand the importance of the time out.'

Participant 3
Another respondent verified this:

'I think it depends on where you are and what area you're working with and what they [the senior medical and nursing staff in the theatre] expect you to do.'

Participant 1

Theme 3: Nurses' perception of their role in conducting the checklist

The final theme that emerged from this study was how nurses perceive their role in conducting the SSC and advocating for patient safety in the operating room.

Interview participants were questioned about their beliefs regarding the role of the operating room nurse in conducting the SSC. A majority of respondents felt that the nurses' role is mainly to confirm patient and procedure identification and existence of the consent form. Only one respondent reported equipment and implant confirmation as part of the nurse role.

'To check the patients name band when the patient is saying their name and also, I guess, to ensure all the details are correct.'

Participant 3

'We participate in the checklist with the doctors and read out the ID band and correlate [sic] all the information on the consent is correct.'

Participant 7

These results are consistent with what was observed during phase 1 where the researcher noted that the nurses who were being observed appeared to be focused primarily on patient identification, procedure and consent aspects of the time out, with little nurse-specific concerns or information.

However, nurses did identify that the checklist enabled them to advocate for and protect the safety of their patients.

'We need to ensure that the patient is protected by communicating with the team.'

Participant 4

Participants were questioned about whether they felt their role was valued by other members of the multidisciplinary team. One participant expressed their frustration that nurses don't appear to be regarded as important members of the team.

'They [surgeons] degrade it because they know we can quickly grab some instruments if we need it, our input is disregarded because it's such a small amount of information we give for the patient and, on the whole, the surgical side is more important.'

Participant 8

Another participant reported a similar experience.

'I don't think we're valued as much as we should [be]. It's basically the doctors doing and speaking to each other, I find. We're just kind of there.'

Participant 6

Another respondent reported that it depended on the team and individuals present at the time.

'It's surgeon and team dependent, sometimes they don't know that you're missing until you push your way into the checklist.'

Participant 7

Other interviewees reported positive experiences with the multidisciplinary team and felt that their input was valued.

'From my experience, in most theatres they appreciate the input.'

Participant 1 
'In the team I work in we all feel like we're listened to and taken seriously.'

Participant 3

The differences in how nurses perceived their role is valued by other members of the operating room team likely reflects the differences in team culture observed within various teams.

\section{Discussion and implications for perioperative nurses}

Although the SSC was introduced as a safety tool to enhance interprofessional communication and prevent surgical errors ${ }^{3}$, this study reports inconsistencies in the behaviours and practices of individuals and groups of surgical teams while conducting the SSC. The differences in team cultures and dynamics that were observed indicate that there are a variety of attitudes and behaviours regarding safety processes which may have implications for patient outcomes. Research into preventable surgical errors link suboptimal team dynamics, time pressures, hierarchical power imbalances, distractions and the transient nature of surgical teams as barriers to effective communication which subsequently contributes to patient harm ${ }^{16-18}$. Studies undertaken of successful SSC implementation in other health care facilities recommend creating an organisational culture of safety. This is defined by Gillespie et $\mathrm{al}^{19}$ as 'an organisational wide commitment to patient safety'. Adopting an organisational safety culture involves using strategies to facilitate effective leadership, communication and interdisciplinary collaboration, and creating a shared mental model for improving patient outcomes 19,20. One such strategy that has been recommended for improving how teams work together in the health care industry is the multidisciplinary team huddle. Team huddles or briefings in the operating room occur at the beginning of the list where staff introduce themselves and discuss anticipated critical events for the day. The team huddle enhances patient safety by enabling the multidisciplinary team to create goals, promote camaraderie and overcome hierarchical power imbalances that limit communication amongst health care teams ${ }^{21-23}$. Another strategy worthy of consideration to address the issue of noise and distractions during the SSC is to adopt the 'sterile cockpit' or 'below ten thousand' concept. This concept was developed as a safety mechanism in the aviation industry and has been adopted within the health care industry. It involves all team members halting nonessential work to avoid distractions and interruptions during critical stages of a patient's care ${ }^{24}$. A small study conducted in the operating room that adopted the below ten thousand rule while conducting the SSC reported this strategy improved multidisciplinary teamwork through increased situational awareness, which allowed the surgical team to focus on the task at hand, improved leadership and promoted the development of high performance teams ${ }^{25}$.

The findings of this study suggest that perioperative nurses are willing to commit to and incorporate the SSC in their everyday practice and value its role in preventing patient harm. However, significant inconsistencies in nursing team participation were observed and there are a variety of beliefs about how operating room nurses should participate while conducting the SSC, which may have implications for patient safety. Critical information shared by other multidisciplinary team members while conducting the SSC may not be communicated to non- participating nurses. Given communication failures within health care teams are identified as a major contributing factor to sentinel and adverse events in the operating room, this is an area that needs improvement? Further education about and training in the requirements for full team participation in conducting the SSC may be required for operating room nurses to overcome any knowledge deficits and improve nurse participation in conducting the SSC.

One of the objectives of this research was to explore nurse's compliance with verifying instrument sterility and equipment availability. The research findings suggest that the role of nurses in conducting the SSC is limited as nurses tend to focus mainly on confirming the correct patient site, side and surgery and provide little nurse-specific information to the multidisciplinary team. Similar findings have also been reported in previous international studies where nurses demonstrated poor compliance with instrument sterility verification and equipment availability ${ }^{26}$. A 2009 study on communication failures between operating room nurses and surgeons suggests that operating room nurses tend to remain silent regarding surgeons' equipment and instrument requirements to avoid appearing incompetent to the rest of the surgical team ${ }^{27}$. Another study suggested that the nurse-specific aspect of the checklist tends to be overlooked as it focuses on latent rather than active risks to patient safety $^{28}$.

The implications of operating room not adhering to the nurse-specific section of the SSC is problematic as ensuring instruments are sterile and surgical equipment is available for procedures is a fundamental responsibility of the operating room nurse ${ }^{27}$. This research has uncovered 
the challenges operating room nurses faced with complying with the nursing review of the checklist. The SSC was conducted as soon as the patient entered the operating room, at a time where instruments trays were not opened. The nurses interviewed reported that it is not appropriate to verify instrument sterility while the checklist is being conducted due to the amount of staff activity around the operating room which is linked with microbial contamination (ACORN 2018).

The ACORN Standards also state that instrument trays should be opened as close as possible to the time of surgery; therefore, simply changing practice by opening sterile instruments before the patient enters the room may not be in the patient's best interest. Modifications in how the checklist is conducted may allow nurses to comply with the instrument sterility verification step. WHO SSC guidelines recommend nurses confirm instrument sterility in the second phase of the SSC, before skin incision. Modifying the checklist into two phases (before induction and before skin incision) rather than just one may provide nurses with enough time to conduct their instrument sterility checks and maintain best practice of not opening instruments too early.

Operating room nurses play an important role as advocate for safe patient outcomes during surgery, as nursing interventions are directed towards prevention of undesirable outcomes such as injury and infection ${ }^{29}$. The findings of this study suggest that attitudes of the multidisciplinary team toward the role of nurses in conducting the SSC have the potential to empower or hinder nurses actively engaging in this process. This research also reveals that while perioperative nurses value the role of the checklist in promoting safe patient outcomes, the role nurses have within the multidisciplinary team tends to be passive and limited. This is concerning as other research findings indicate that nurses are less likely to speak up about any patient safety concerns when there is poor collaboration with medical staff $^{30}$. Strategies aimed to improve communication between medical and nursing staff by creating a culture of safety, as discussed earlier, may facilitate nurses to better engage with the multidisciplinary team and enhance their role in conducting the SSC.

\section{Limitations}

This study was conducted as part of the primary researcher's research dissertation and there were associated time restraints. This was a small-scale project conducted on a single site, involved one researcher and was based on data collected from relatively limited observation and interview participants; therefore, it is not transferable to other health care settings. Focused ethnographic research is subjective in nature and the interpretative skills of the primary researcher can impact on the trustworthiness of the findings ${ }^{12}$. Although methods such as maintaining a field journal were used to promote objectivity, the primary researcher's high degree of familiarity with the participants and setting may have introduced preconceived assumptions to the research. It is also possible that a Hawthorne effect may have distorted the observational data as staff were aware that they were being observed during the checklist ${ }^{8}$. Key informants were restricted to operating room nurses only. Perspectives from other disciplines such as anaesthetic nurses and medical staff may have enriched the research data by providing additional insight into the expectations of operating room nurses about conducting the SSC.

\section{Conclusions}

This study provides a glimpse of how operating room nurses engage with the SSC within an Australian context. The findings on barriers to team compliance with conducting the checklist are consistent with previous research undertaken in Australian settings. The themes identified from this research highlight different factors that influence how nurses engage with the multidisciplinary team while the SSC is conducted. Strategies aimed at improving the functioning of surgical teams through promotion of a culture of safety, fostering better leadership and enabling staff to effectively communicate with one another may be beneficial in overcoming the poor team dynamics that impact on compliance with conducting the sSC. The nurse-specific role in the checklist process is an essential component of patient safety as it addresses the risk for surgical delays and surgical site infections. Empowering nurses to have an active role within the multidisciplinary team and modifying current checklist practices may be required to encourage operating room nurses to better engage with the nurse-specific area of the SSC.

\section{Knowledge translation}

- Creating a culture of safety within the multidisciplinary team is essential for high-performing teams to successfully implement the SSC.

- Perioperative nurses play an important role in promoting safe patient outcomes which needs to be recognised by the multidisciplinary team. All members of the operating room nursing team should participate in the SSC to ensure they are all aware of information relating to patient safety. 
- Strategies to empower operating room nurses to engage with the nurse-specific section of the SSC and verifying instrument sterility and equipment availability are necessary to overcome poor compliance.

\section{References}

1. Haynes AB, Weiser TG, Berry WR, Lipsitz SR, Breizat AH, Dellinger EP et al. A surgical safety checklist to reduce morbidity and mortality in a global population. N Engl J Med 2009;360(5):491-499.

2. Zegers $M$, de Bruijne $M$, de Keizer $B$, Merten $\mathrm{H}$, Groenewegen PP, van der Wal $\mathrm{G}$ et al. The incidence, root-causes, and outcomes of adverse events in surgical units: Implication for potential prevention strategies. Patient Saf Surg 2011;5:13.

3. Treadwell JR, Lucas S, Tsou AY. Surgical checklists: A systematic review of impacts and implementation. BMJ Qual Saf 2014;23(4):299-318.

4. World Health Organization (WHO). Implementation manual surgical safety checklist [Internet]. Geneva: WHO; 2009 [cited 2019 Oct 12]. Available from: apps.who.int/iris/bitstream/ handle/10665/44186/9789241598590_eng. pdf;sequence=1.

5. O'Connor P, Reddin C, O'Sullivan M, O'Duffy F, Keogh I. Surgical checklists: The human factor. Patient Saf Surg 2013;7(1):14.

6. Australian College of Perioperative Nurses Ltd (ACORN). Standards for Perioperative Nursing in Australia $15^{\text {th }}$ ed. Adelaide, South Australia: ACORN; 2018.

7. Braaf S, Manias E, Riley R. The 'time-out' procedure: An institutional ethnography of how it is conducted in actual clinical practice. BMJ Qual Saf 2013 Aug;22(8):647-655.

8. Gillespie BM, Harbeck EL, Lavin J. Evaluation of a patient safety program on surgical safety checklist compliance: a prospective longitudinal study. BMJ Open Quality 2018;7:e000362. doi: 10.1136/ bmjoq-2018-000362.
9. Gillespie B. Impact of workflow on the use of the Surgical Safety Checklist: A qualitative study. ANZ J Surg 2016;86(1):864-867.

10. Schneider Z. Whitehead D. Elliott D.LoBiondo-Wood G. Haber J. Nursing and midwifery research: Methods and appraisal for evidence-based practice. $5^{\text {th }}$ ed. NSW: Elsevier, 2016.

11. Cruz E, Higginbottom G. The use of focused ethnography in nursing research. Nurse Res 2013;20(4):36-43.

12. Roper JM, Shapira J. Methods in nursing research: Ethnography in nursing research. Thousand Oaks, CA: SAGE Publications; 2000.

13. Braun V, Clarke V. Using thematic analysis in psychology. Qual Res Psychol 2006;3(1):77101. Available from: www.scribd.com doc/151684840/Braun-Clarke-2006-UsingThematic-Analysis.

14. McBrien B. Evidence-based care: Enhancing the rigour of a qualitative study. $\mathrm{Br}$ J Nurs 2008;17(20):1286-1289.

15. Nowell LS, Norris JM, White DE, Moules NJ. Thematic analysis: Striving to meet the trustworthiness criteria. Int J Qual Methods 2017. Available from: doi.org/10.1177/1609406917733847.

16. Gillespie BM, Chaboyer W, Murray P. Enhancing communication in surgery through team training interventions: A systematic literature review. AORN J 2010;92(6):642-657.

17. Leach LS, Myrtle RC, Weaver FA. Surgical teams: Role perspectives and role dynamics in the operating room. Health Serv Manage Res 2011;24(2):81-90.

18. Rhee AJ, Valentin-Salgado Y, Eshak D, Feldman D, Kischak P, Reich DL et al. Team training in the perioperative arena: A methodology for implementation and auditing behavior. Am J Med Qual 2017;32(4):369-375.

19. Gillespie BM, Gwinner K, Chaboyer W, Fairweather $\mathrm{N}$. Team communications in surgery - creating a culture of safety. J Interprof Care 2013;27(5):387-393.
20. Wilson A. Creating and applying shared mental models in the operating room. Journal of Perioperative Nursing 2019;32(3):33-36.

21. Landers R. Reducing surgical errors: Implementing a three-hinge approach to success. AORN J 2015;101(6):657-665.

22. Jones KC, Ritzman T. Perioperative safety: Keeping our children safe in the operating room. Orthop Clin North Am 2018 49(4):465476.

23. White BAA, Eklund A, McNeal T, Hochhalter A, Arroliga AC. Facilitators and barriers to ad hoc team performance. Proc (Bayl Univ Med Cent) 2018;31(3):380-384.

24. Kapur N, Parand A, Soukup T, Reader T, Sevdalis N. Aviation and healthcare: A comparative review with implications for patient safety. JRSM Open 2016;7(1):2054270415616548.

25. Smith P, Gibbs J. 'Below ten thousand': An effective behavioural noise reduction strategy? Journal of Perioperative Nursing 2016;29(3):29-32.

26. Putnam LR, Levy SM, Sajid M, Dubuisson DA, Rogers NB, Kao LS et al. Multifaceted interventions improve adherence to the surgical checklist. Surgery 2014;156(2):336344. Available from: www.sciencedirect.com/ science/article/pii/\$0039606014001263.

27. Gardezi F, Lingard L, Espin S, Whyte S, Orser, Baker GR. Silence, power and communication in the operating room. J Adv Nurs 2009;65(7):1390-1399. doi:10.1111 /j.1365-2648.2009.04994.

28. Rydenfält $C$, Johansson $G$, Odenrick P, Akerman K, Larsson PA, Åkerman K. Compliance with the WHO surgical safety checklist: Deviations and possible improvements. Intl J Qual Health Care 2013;25(2):182-187.

29. Rothrock JC, McEwen D. Alexander's Care of the Patient in Surgery. $12^{\text {th }}$ ed. St. Louis: Mosby; 2018.

30. Carney BT, West P, Neily J, Mills PD, Bagian JP. Differences in nurse and surgeon perceptions of teamwork: Implications for use of a briefing checklist in the OR. AORN J 2010 Jun;91(6):722-729. 